\title{
ON THE CONVERGENCE OF EXTREME DISTRIBUTIONS UNDER POWER NORMALIZATION
}

Abstract. This paper deals with the convergence in distribution of the maximum of $n$ independent and identically distributed random variables under power normalization. We measure the difference between the actual and asymptotic distributions in terms of the double-log scale. The error committed when replacing the actual distribution of the maximum under power normalization by its asymptotic distribution is studied, assuming that the cumulative distribution function of the random variables is known. Finally, we show by examples that the convergence to the asymptotic distribution may not be uniform in this double-log scale.

1. Introduction. Let $X_{1}, \ldots, X_{n}$ be independent random variables with common distribution function (d.f.) $F(x)=P\left(X_{n} \leq x\right)$, and $X_{1: n} \leq \cdots \leq$ $X_{n: n}$ be the corresponding order statistics. Suppose there exist norming constants $\alpha_{n}, \beta_{n} \in \mathbb{R}$ and a d.f. $K(x)$ such that as $n \rightarrow \infty$,

$$
F^{n}\left(\alpha_{n} x+\beta_{n}\right) \stackrel{w}{\rightarrow} K(x),
$$

where $\stackrel{w}{\rightarrow}$ denotes weak convergence. We call $K$ a max stable d.f. under linear normalization or simply an $\ell$-max stable law. It is well known that an $\ell$-max stable law can only be one of Gnedenko's extreme value distributions (see [1], [7], [10]). If (1) holds, we write $F \in D_{\ell}(K)$ to indicate that $F$ belongs to the maximal domain of attraction of $K$ under linear normalization. In order to improve the accuracy of the approximation of the distribution of the maximum for large values of $n,[13,15]$ introduced a nonlinear normalization called power normalization. Following $[12,13]$, a d.f. $F$ is said to belong to the maximal domain of attraction of a d.f. $H(x)$ under power normalization

2000 Mathematics Subject Classification: 60F05, 62F15, 62E20, 62 G30.

Key words and phrases: power normalization, $p$-max stable laws, relative risk, double$\log$ scale, uniformity of convergence. 
if there exist norming constants $a_{n}, b_{n}>0$ such that, as $n \rightarrow \infty$,

$$
P\left(\left|\frac{X_{n: n}}{a_{n}}\right|^{1 / b_{n}} \mathcal{S}\left(X_{n: n}\right) \leq x\right)=F^{n}\left(a_{n}|x|^{b_{n}} \mathcal{S}(x)\right) \stackrel{w}{\rightarrow} H(x),
$$

where $\mathcal{S}(x)=-1 ; 0 ; 1$ if respectively $x<0 ; x=0 ; x>0$. In this case we write $F \in D_{p}(H)$. We call $H(x)$ a max stable d.f. under power normalization or simply a $p$-max stable d.f. if (2) holds. We say that two d.f.'s $F_{1}$ and $F_{2}$ are of the same $p$-type if there exist $A, B>0$ such that $F_{1}(x)=F_{2}\left(A|x|^{B} \mathcal{S}(x)\right)$ for all $x$. [13] showed that any $p$-max stable d.f. $H(x)$ has the $p$-type of one of the six d.f.'s $H_{\beta}^{(i)}(x)=\exp \left(-\mathcal{V}_{i ; \beta}(x)\right), i=1, \ldots, 6, \beta>0$, where

$$
\begin{aligned}
& \text { Types I : } \mathcal{V}_{1 ; \beta}(x)= \begin{cases}\infty, & x \leq 1, \\
(\ln x)^{-\beta}, & x>1 ;\end{cases} \\
& \text { Types II }: \mathcal{V}_{2 ; \beta}(x)= \begin{cases}\infty, & x \leq 0, \\
(-\ln x)^{\beta}, & 0<x \leq 1, \\
0, & x>1 ;\end{cases} \\
& \text { Types III : } \mathcal{V}_{3 ; \beta}(x)= \begin{cases}\infty, & x \leq-1, \\
(-\ln (-x))^{-\beta}, & -1<x \leq 0, \\
0, & x>0 ;\end{cases}
\end{aligned}
$$

$$
\begin{aligned}
& \text { Types IV }: \quad \mathcal{V}_{4 ; \beta}(x)= \begin{cases}(\ln (-x))^{\beta}, & x \leq-1, \\
0, & x>-1 ;\end{cases} \\
& \text { Type V }: \quad \mathcal{V}_{5 ; \beta}(x)=\mathcal{V}_{5}(x)= \begin{cases}\infty, & x \leq 0, \\
1 / x, & x>0 ;\end{cases} \\
& \text { Type VI }: \quad \mathcal{V}_{6 ; \beta}(x)=\mathcal{V}_{6}(x)= \begin{cases}-x, & x<0, \\
0, & x \geq 0 .\end{cases}
\end{aligned}
$$

Necessary and sufficient conditions for a d.f. to belong to $D_{p}(\cdot)$ for each of the six $p$-max stable laws were obtained in $[12,14]$. In these papers the results of $[8,9]$ concerning linear normalization were extended to $p$-max stable laws. They showed that every d.f. attracted to an $\ell$-max stable law is necessarily attracted to some $p$-max stable law and that $p$-max stable laws, in fact, attract more. For more details on power normalization see [2]-[5].

The normalizing constants $a_{n}$ and $b_{n}$ can be chosen as follows (see [12]):

$$
\begin{array}{lll}
F \in D_{p}\left(\exp \left(-\mathcal{V}_{1 ; \beta}(x)\right)\right): & a_{n}=1, & b_{n}=\ln F^{-}(1-1 / n), \\
F \in D_{p}\left(\exp \left(-\mathcal{V}_{2 ; \beta}(x)\right)\right): & a_{n}=x^{0}, & b_{n}=\ln \left[\frac{x^{0}}{F^{-}(1-1 / n)}\right], \\
F \in D_{p}\left(\exp \left(-\mathcal{V}_{3 ; \beta}(x)\right)\right): & a_{n}=1, & b_{n}=-\ln \left[-F^{-}(1-1 / n)\right],
\end{array}
$$




$$
\begin{array}{lll}
F \in D_{p}\left(\exp \left(-\mathcal{V}_{4 ; \beta}(x)\right)\right): & a_{n}=-x^{0}, & b_{n}=\ln \left[\frac{F^{-}(1-1 / n)}{x^{0}}\right], \\
F \in D_{p}\left(\exp \left(-\mathcal{V}_{5 ; \beta}(x)\right)\right): & a_{n}=F^{-}(1-1 / n), & b_{n}=f\left(a_{n}\right), \\
F \in D_{p}\left(\exp \left(-\mathcal{V}_{6 ; \beta}(x)\right)\right): & a_{n}=-F^{-}(1-1 / n), & b_{n}=f\left(-a_{n}\right),
\end{array}
$$

where $F^{-}(y)=\inf \{x: F(x)>y\}, x^{0}=\sup \{x: F(x)<1\}$ and

$$
f(t)=(1-F(t))^{-1} \int_{t}^{x^{0}} \frac{1-F(x)}{x} d x .
$$

For these results to be useful in practice one needs to know the order of magnitude of the error committed when the actual cdf $F^{n}\left(a_{n}|x|^{b_{n}} S(x)\right)$ is replaced by the asymptotic cdf $H_{\beta}^{(i)}(x)$. One way of measuring this error is provided by the arithmetical scale of probabilities which leads to the absolute error $F^{n}\left(a_{n}|x|^{b_{n}} S(x)\right)-H_{\beta}^{(i)}(x)$. In addition, the double-log scale and inverse scale that leads to the idea of "return period" may be used to measure the error. The speed of convergence of the distribution of the maximum of a sample of independent and identically distributed random variables under linear normalization to its asymptotic distribution is considered in [11]. In this paper the convergence of the distribution of the maximum of a sample of independent and identically distributed random variables under power normalization is studied. The practical importance of the double-log scale is considered in Section 2. The order of magnitude of the error in the double-log scale and the uniformity of the convergence are studied in Section 3.

2. The double-log scale property. Many properties of probability distributions can be expressed adequately using the arithmetical scale. However, there are practical situations when the occurrence of an event relating to the distribution of the maximum or minimum has a catastrophic character. In these cases, one typically wants to evaluate the risk incurred because of taking a decision. When a cdf $H(x)$ takes values in a small neighborhood of 1 , the arithmetical scale makes it difficult to perceive and to represent graphically the often significant consequences of a seemingly small change in $H(x)$. So, the double-log scale to measure the error deserves consideration. In this scale we use $\left|\zeta_{F}-\zeta_{H}\right|$, where

$$
\zeta_{F}=-\ln \left\{-\ln F^{n}\left(a_{n}|x|^{b_{n}} S(x)\right)\right\} \quad \text { and } \quad \zeta_{H}=-\ln \{-\ln H(x)\} .
$$

Also, we can use the inverse scale or "return period" defined by $\delta_{H}=$ $(1-H(x))^{-1}$, which gives the expected number of observations of the random variable required to obtain the first observation larger than quantile of $H(x)$. According to these scales, a probability $H(x)=1-(\ln a)^{-1}$ may be represented by $\xi_{H}=-\ln \left(-\ln \left(1-(\ln a)^{-1}\right)\right) \approx \ln \ln a$ or $\delta_{H}=\ln a$, the approximation being valid if $H(x)$ is close to 1 . When $a=10$, the corre- 
sponding probability is represented in terms of the arithmetical, double-log, and inverse scales by $0.0,0.0$ and 1.0 , respectively. If a decision is taken to choose $a=100$, these figures must be replaced by $0.5,0.3$ and 2 . While the difference is 0.005 in the arithmetical scale, it is 0.003 in the double-log scale, and 0.001 for the return period. The relative risk is 2 , and the $\ln$ of the relative risk is 0.30 , so the difference between the double-log values is almost exactly equal to the $\ln$ of the relative risk.

In the double-log scale, the equations $y=H_{\beta}^{(i)}(x), i=1, \ldots, 6$, represent curves in the coordinates $(x, y)$, but become straight lines in the coordinates $(\xi, \eta)$, where $\xi=-\ln \left(-\ln H_{\beta}^{(i)}(x)\right)$ and $\eta=\ln \ln x$ for $i=1, \eta=-\ln (-\ln x)$ for $i=2, \eta=-\ln (-\ln (-x))$ for $i=3, \eta=-\ln \ln (-x)$ for $i=4, \eta=\ln x$ for $i=5$ and $\eta=-\ln (-x)$ for $i=6$.

3. Uniformity of convergence. The convergence of $F^{n}\left(a_{n}|x|^{b_{n}} S(x)\right)$ to $H_{\beta}^{(i)}(x)$ and the continuity of the double-log scale transformation imply the convergences of $\xi_{F}$ to $\xi_{H}$. If these convergence were uniform, the error committed using $H_{\beta}^{(i)}(x)$ instead of $F^{n}\left(a_{n}|x|^{b_{n}} S(x)\right)$ would be bounded in the upper tail when using the double-log scale. The uniformity is an important property in practical applications. We now give two theorems to show that the convergence is not always uniform in the double-log scale and the greater the sample size $n$, the smaller the bound would be.

TheOREM 3.1. Let $F(x)$ be absolutely continuous and suppose $G(z x)=$ $z^{-\beta}\left(1-F\left(x^{0} \exp (-z x)\right)\right)$ is finite and has a limit $M \neq 0$ as $z \rightarrow 0$. Then $F(x)$ is in the domain of attraction of $H_{\beta}^{(2)}(x)$ and the convergence in the upper tail is uniform in $x$ in the double-log scale.

Proof. $F(x) \in D_{P}\left(H_{\beta}^{(2)}(x)\right)$ if and only if (see [12])

$$
\lim _{z \rightarrow 0} \frac{1-F\left(x^{0} \exp (-z x)\right)}{1-F\left(x^{0} \exp (-z)\right)}=x^{\beta}, \quad x, \beta>0 .
$$

Since $G(z)$ has a limit $M \neq 0$, we have

$$
\lim _{z \rightarrow 0} \frac{1-F\left(x^{0} \exp (-z x)\right)}{1-F\left(x^{0} \exp (-z)\right)}=\lim _{z \rightarrow 0} \frac{G(z x)}{G(z)} x^{\beta}=x^{\beta},
$$

and therefore $F(x)$ is in the domain of attraction of $H_{\beta}^{(2)}(x)$.

Let $h(z, x)=G(z x) / G(z)$. Then $\lim _{z \rightarrow 0} h(z, x)=1, x>0$. Choosing $a_{n}=x^{0}, F\left(x^{0} \exp \left(-b_{n}\right)\right)=1-1 / n, S(x)=1$ one obtains

$$
\begin{aligned}
\xi_{F} & =-\ln \left(-n \ln F\left(x^{0}|x|^{b_{n}}\right)\right) \\
& =-\ln \left(-n \ln \left(1-(-\ln x)^{\beta} h\left(b_{n},-\ln x\right)\right)\left(1-F\left(x^{0} \exp \left(-b_{n}\right)\right)\right)\right)
\end{aligned}
$$




$$
\begin{aligned}
\approx & \left.\left.-\ln \left(n(-\ln x)^{\beta} h\left(b_{n},-\ln x\right)\right)\right)\left(1-F\left(x^{0} \exp \left(-b_{n}\right)\right)\right)\right) \\
= & -\ln n-\beta \ln (-\ln x)-\ln \left(h\left(b_{n},-\ln x\right)\right) \\
& -\ln \left(1-F\left(x^{0} \exp \left(-b_{n}\right)\right)\right) \\
= & -\beta \ln (-\ln x)-\ln \left(h\left(b_{n},-\ln x\right)\right),
\end{aligned}
$$

where $0<x<1$ and the approximation is valid when $x$ is close to 1 . Moreover,

$$
\xi_{H_{\beta}^{(2)}}=-\ln \left(-\ln \left(\exp \left(-(-\ln x)^{\beta}\right)\right)\right)=-\beta \ln (-\ln x) .
$$

Then

$$
\Delta=\left|\xi_{F}-\xi_{H_{\beta}^{(2)}}\right|=\left|\ln \left(h\left(b_{n},-\ln x\right)\right)\right| .
$$

Therefore, the convergence is uniform in $x$ in the upper tail if $h\left(b_{n},-\ln x\right)$ can be bounded in the neighborhood $0<x<\varepsilon$ of zero.

Finally, because $\lim _{z \rightarrow 0} G(z)=M$ and $\lim _{z \rightarrow 0} G(x z)=M$, for any $\varepsilon>0$ we have

$$
1-\frac{2 \varepsilon}{M}<h(z, x)<\frac{M+\varepsilon}{M-\varepsilon}<1+\frac{4 \varepsilon}{M},
$$

the last inequality being valid if $\varepsilon<M / 2$. Hence if $\varepsilon$ is small, then we have $\left|\ln \left(h\left(b_{n},-\ln x\right)\right)\right|<\frac{4 \varepsilon}{M} \ln e$ for any $b_{n}>0$ and $0<x<1$, and consequently the convergence to $H_{\beta}^{(2)}(x)$ is uniform in $x$ in the upper tail when the double$\log$ scale is used.

TheOREM 3.2. Let $F(x) \in D_{P}\left(H_{\beta}^{(2)}(x)\right)$ and $\lim _{z \rightarrow 0} G(z)=M$, where $G(z)=\left(1-F\left(x^{0} \exp (-z)\right)\right) z^{-\beta}$ and $M=0$ or $\infty$. Then the convergence is non-uniform in $x$ in the double-log scale. Moreover, for any $\alpha>0$, one has $\lim _{z \rightarrow 0} G(z) z^{-\alpha}=\infty$ if $M=0$, and $\lim _{z \rightarrow 0} G(z) z^{\alpha}=0$ if $M=\infty$.

Proof. If $\lim _{z \rightarrow 0} G(z)=0$ and $F(x) \in D_{P}\left(H_{\beta}^{(2)}(x)\right)$, then the rate of convergence of $G(z)$ to 0 must be compatible with the necessary and sufficient condition for $F(x)$ to be in the domain of attraction of $H_{\beta}^{(2)}(x)$, which is

$$
\lim _{z \rightarrow 0} \frac{G(z x)}{G(z)}=1, \quad x>0 .
$$

If $\lim _{z \rightarrow 0} G(z) z^{-\alpha}=M>0$, one has

$$
\lim _{z \rightarrow 0} \frac{G(z x)}{G(z)}=\lim _{z \rightarrow 0} \frac{(z x)^{-\alpha} G(z x)}{z^{-\alpha} G(z)} x^{\alpha}=x^{\alpha} \neq 1,
$$

and therefore $F(x)$ is not in the domain of attraction of $H_{\beta}^{(2)}(x)$. Similarly, when $\lim _{z \rightarrow 0} G(z)=\infty$ and $F(x) \in D_{P}\left(H_{\beta}^{(2)}(x)\right)$. 
REMARK 3.1. Theorem 3.1 provides conditions for $F^{n}\left(a_{n}|x|^{b_{n}} S(x)\right)$ to converge uniformly to $H_{\beta}^{(2)}(x)$, while Theorem 3.2 gives conditions under which the convergence to $H_{\beta}^{(2)}(x)$ is non-uniform. The most important consequence of these theorems is that the convergence to $H_{\beta}^{(2)}(x)$ and $H_{\beta}^{(i)}(x)$, $i=1,3,4,5,6$, must be uniform or, otherwise, the difference $\left|\xi_{F}-\xi_{H_{\beta}^{(i)}}\right|$ is at most a logarithmic function of $\eta$, where $\eta=\ln \ln x$ for $i=1, \eta=-\ln (-\ln x)$ for $i=2, \eta=-\ln (-\ln (-x))$ for $i=3, \eta=-\ln \ln (-x)$ for $i=4, \eta=\ln x$ for $i=5$ and $\eta=-\ln (-x)$ for $i=6$.

EXAMPLE 3.1. Let

$$
F(x)= \begin{cases}1, & x>1, \\ 1-(-\ln x)^{\beta}(-\ln (-\ln x))^{M}, & a<x \leq 1, \\ 0, & x \leq a,\end{cases}
$$

where $\beta>0, M$ is a given real number and $a$ satisfies $a \geq \exp (-1), a \geq$ $\exp (-\exp (-M / \beta))$, and $a \geq x^{*}$ for every solution $x^{*}$ of the equation $1-$ $\left(-\ln \left(x^{*}\right)\right)^{\beta}\left(-\ln \left(-\ln \left(x^{*}\right)\right)\right)^{M}=0$ in the interval $(0,1)$. Since

$$
\lim _{z \rightarrow 0} \frac{1-F\left(x^{0} \exp (-z x)\right)}{1-F\left(x^{0} \exp (-z)\right)}=x^{\beta}, \quad x^{0}=1,
$$

we have $F(x) \in D_{P}\left(H_{\beta}^{(2)}(x)\right)$ with $a_{n}=1$ and $b_{n}^{\beta}\left(-\ln b_{n}\right)^{M}=1 / n$, and when $S(x)=1$,

$$
\begin{aligned}
\xi_{F} & =-\ln \left(-n \ln F\left(a_{n}|x|^{b_{n}}\right)\right) \\
& =-\ln \left(-n \ln \left(1-\left(-\ln \left(x^{b_{n}}\right)\right)^{\beta}\left(-\ln \left(-\ln \left(x^{b_{n}}\right)\right)\right)^{M}\right)\right) \\
& =-\ln \left(-n \ln \left(1-b_{n}^{\beta}(-\ln x)^{\beta}\left(-\ln \left(b_{n}(-\ln x)\right)\right)^{M}\right)\right) \\
& =-\ln \left(-n \ln \left(1-\frac{(-\ln x)^{\beta}}{n}\left(\frac{-\ln b_{n}-\ln (-\ln x)}{-\ln b_{n}}\right)^{M}\right)\right) \\
& \left.\approx-\ln \left((-\ln x)^{\beta}\left(\frac{-\ln b_{n}-\ln (-\ln x)}{-\ln b_{n}}\right)^{M}\right)\right) \\
& =-\beta \ln (-\ln x)-M \ln \left(\frac{-\ln b_{n}-\ln (-\ln x)}{-\ln b_{n}}\right),
\end{aligned}
$$

where the approximation is valid for any $0<x<1$ close to one. Also,

$$
\xi_{H_{\beta}^{(2)}}=-\beta \ln (-\ln x) .
$$

Hence

$$
\Delta=\left|\xi_{F}-\xi_{H_{\beta}^{(2)}}\right|=\left|M \ln \left(\frac{-\ln b_{n}-\ln (-\ln x)}{-\ln b_{n}}\right)\right|,
$$


which is an unbounded function of $\eta=-\ln (-\ln x)$ when $\eta$ tends to infinity provided that $M \neq 0$, and obviously tends to 0 as $n$ tends to infinity for each given $\eta$. Hence the convergence of $\xi_{F}$ to $\xi_{H_{\beta}^{(2)}}$ is uniform in $x$ if $M=0$ but is non-uniform in $x$ if $M \neq 0$.

EXAmPle 3.2. Let $F(x)=1-\exp \left(-(\ln (-x))^{-\beta}\right), x \leq-1$. In order to study the uniformity of the convergence in the double-log scale one has to analyze the difference $\left|\xi_{F}-\xi_{H}\right|$, where in the case $S(x)=1$,

$$
\begin{aligned}
\xi_{F} & =-\ln \left(-n \ln \left(1-\exp \left(-\left(\ln \left(-a_{n}|x|^{b_{n}}\right)\right)^{-\beta}\right)\right)\right) \\
& =-\ln \left(n \exp \left(-\left(\ln \left(-a_{n}|x|^{b_{n}}\right)\right)^{-\beta}\right)\right) \\
& \approx-\ln n+\left(\ln \left(-a_{n}|x|^{b_{n}}\right)\right)^{-\beta} \ln e,
\end{aligned}
$$

where the approximation is valid for large values of $x$, and

$$
\xi_{H_{\beta}^{(4)}}=-\ln \left(-\ln \left(\exp \left(-(\ln (-x))^{\beta}\right)\right)\right)=-\ln \left((\ln (-x))^{\beta}\right) .
$$

Hence

$$
\begin{aligned}
\Delta_{1} & =\left|\xi_{F}-\xi_{H_{\beta}^{(4)}}\right| \\
& =\left|-\ln n+\left(\ln \left(-a_{n}|x|^{b_{n}}\right)\right)^{-\beta} \ln e+\beta \ln \ln (-x)\right| .
\end{aligned}
$$

For fixed $n$, this expression is an unbounded function of $x$ for any $\beta>0$, except for $\beta=1$ with $b_{n}=1$. Therefore, the convergence of $F^{n}\left(\alpha_{n}|x|^{\beta_{n}} S(x)\right)$ to $H_{\beta}^{(4)}$ is non-uniform in $x$ in the double-log scale for any $\beta \neq 1$, and the difference $\Delta_{1}$ in the upper tail is a logarithmic function of $\eta=-\ln \ln (-x)$ with exponent $\beta$.

ExAmPle 3.3. Let $F(x)=1-\exp \left(-(-\ln (-x))^{\beta}\right),-1<x<0$. Then

$$
\begin{aligned}
\xi_{F} & =-\ln \left(-n \ln \left(1-\exp \left(-\left(-\ln \left(-a_{n}|x|^{b_{n}}\right)\right)^{\beta}\right)\right)\right) \\
& =-\ln \left(n \exp \left(-\left(-\ln \left(-a_{n}|x|^{b_{n}}\right)\right)^{\beta}\right)\right) \\
& \approx-\ln n+\left(-\ln \left(-a_{n}|x|^{b_{n}}\right)\right)^{\beta} \ln e, \\
\xi_{H_{\beta}^{(3)}} & =-\ln \left(-\ln \left(\exp \left(-(-\ln (-x))^{-\beta}\right)\right)\right) \\
& =-\ln \left((-\ln (-x))^{-\beta}\right) .
\end{aligned}
$$

Hence

$$
\Delta_{2}=\left|-\ln n+\left(-\ln \left(-a_{n}|x|^{b_{n}}\right)\right)^{\beta} \ln e-\beta \ln (-\ln (-x))\right| .
$$

For fixed $n$, this expression is an unbounded function of $x$ for any $\beta>0$, except for $\beta=1$ with $b_{n}=1$. Therefore, the convergence of $F^{n}\left(\alpha_{n}|x|^{\beta_{n}} S(x)\right)$ to $H_{\beta}^{(3)}$ is non-uniform in $x$ in the double-log scale for any $\beta \neq 1$, and the difference $\Delta_{2}$ in the upper tail is a logarithmic function of $\eta=-\ln (-\ln (-x))$ with exponent $-\beta$. 
EXAMPLE 3.4. Let $F(x)=1-\exp \left(-(\ln x)^{\beta}\right), x>1$. Then

$$
\begin{aligned}
\xi_{F} & =-\ln \left(-n \ln \left(1-\exp \left(-\left(\ln \left(a_{n}|x|^{b_{n}}\right)\right)^{\beta}\right)\right)\right) \\
& =-\ln \left(n \exp \left(-\left(\ln \left(a_{n}|x|^{b_{n}}\right)\right)^{\beta}\right)\right) \\
& \approx-\ln n+\left(\ln \left(a_{n}|x|^{b_{n}}\right)\right)^{\beta} \ln e \\
\xi_{H_{\beta}^{(1)}} & =-\ln \left(-\ln \left(\exp \left(-(\ln x)^{-\beta}\right)\right)\right) \\
& =-\ln \left((\ln x)^{-\beta}\right) .
\end{aligned}
$$

Hence

$$
\Delta_{3}=\left|-\ln n+\left(\ln \left(a_{n}|x|^{b_{n}}\right)\right)^{\beta} \ln e-\beta \ln \ln x\right| .
$$

For fixed $n$, this expression is an unbounded function of $x$ for any $\beta>0$, except for $\beta=1$ with $b_{n}=1$. Therefore, the convergence of $F^{n}\left(\alpha_{n}|x|^{\beta_{n}} S(x)\right)$ to $H_{\beta}^{(1)}$ is non-uniform in $x$ in the double-log scale for any $\beta \neq 1$, and the difference $\Delta_{3}$ in the upper tail is a logarithmic function of $\eta=\ln \ln x$ with exponent $\beta$.

It is interesting that, because $\left|\xi_{F}-\xi_{H_{\beta}^{(1)}}\right|$ is a logarithmic function of $\eta$ in the first and second example, the difference between $\xi_{F}$ and $\xi_{H}$ increases very slowly when $\eta$ tends to infinity.

\section{References}

[1] B. C. Arnold, H. Balakrishnan, and H. N. Nagaraja, A First Course in Order Statistics, Wiley, 1992.

[2] H. M. Barakat and E. M. Nigm, Extreme order statistics under power normalization and random sample size, Kuwait J. Sci. Engrg. 29 (2002), 27-41.

[3] - - - Some asymptotic stability properties of extreme order statistics under power normalization, ibid. 29 (2003), 15-27.

[4] H. M. Barakat, E. M. Nigm and E. M. El-Adll, Continuation theorems of the extremes under power normalization, J. Appl. Math. Comput. 10 (2002), 1-15.

[5] - , - - , . Asymptotic properties of random extremes under general normalization from non-identical distributions, Metrika 29 (2004), 275-287.

[6] G. Christoph and M. Falk, A note on domains of attraction of p-max stable laws, Statist. Probab. Lett. 28 (1996), 279-284.

[7] J. Galambos, The Asymptotic Theory of Extreme Order Statistics, Krieger, Malabar, FL, 1987.

[8] B. V. Gnedenko, Sur la distribution limite du terme maximum d'une série aléatoire, Ann. of Math. 44 (1943), 423-453.

[9] L. de Haan, A form of regular variation and its application to the domain of attraction of the double exponential distribution, Z. Wahrsch. Verw. Gebiete 17 (1971), $241-258$.

[10] M. R. Leadbetter, G. Lindgren and H. Rootzén, Extremes and Related Properties of Random Sequences and Processes, Springer, 1983.

[11] A. Luceño, Speed of convergence to the extreme value distributions of their probability plotting papers, Comm. Statist. Simul. 23 (1994), 529-545. 
[12] N. R. Mohan and S. Ravi, Max domains of attraction of univariate and multivariate p-max stable laws, Theory Probab. Appl. 37 (1992), 632-643.

[13] E. Pantcheva, Limit theorems for extreme order statistics under nonlinear normalization, in: Lecture Notes in Math. 1155, Springer, 1985, 284-309.

[14] U. R. Subramanya, On max domains of attraction of univariate p-max stable laws, Statist. Probab. Lett. 19 (1994), 271-279.

[15] S. B. Weinstein, Theory and application of some classical and generalized asymptotic distributions of extreme values, IEEE Trans. Information Theory IT-19 (1973), 148154 .

Department of Mathematics

Faculty of Science

Zagazig University

Zagazig, Egypt

E-mail: s_nigm@yahoo.com

Received on 14.1.2008;

revised version on 19.6.2008 
